\title{
Statistical study of inverted-V structures in FAST data
}

\author{
N. Partamies ${ }^{1}$, E. Donovan ${ }^{2}$, and D. Knudsen ${ }^{2}$ \\ ${ }^{1}$ Finnish Meteorological Institute, Helsinki, Finland \\ ${ }^{2}$ Institute for Space Research, University of Calgary, Calgary, Canada
}

Received: 8 January 2008 - Revised: 21 March 2008 - Accepted: 16 April 2008 - Published: 11 June 2008

\begin{abstract}
Since the 1970s the inverted-V structures have been observed in the satellite-borne particle data. These $\Lambda$ shaped enhancements of the differential energy flux on the high energy channels on the top of low number flux on the lower energies are traces of auroral particle acceleration by U-shaped potential drops along the field lines within the auroral zones. In this study, we show the results of a statistical study of inverted-V events in five years (1997-2001) of the Fast Auroral SnapshoT (FAST) electron spectrograms. We found 588 well-defined events in total, but the statistical results are also shown for a subset of 97 very best events.

The statistical analysis shows that the inverted-V's mainly occur within the auroral oval latitudes $\left(65^{\circ}-75^{\circ}\right.$ invariant latitude), in the pre-midnight sector (21-23 magnetic local time) and during the Northern Hemisphere winter months (November to February). The typical maximum energies of the inverted-V's are $2-4 \mathrm{keV}$, when a threshold value of $0.3 \mathrm{keV}$ is used as a selection criterion. This corresponds to an acceleration potential drop of a few $\mathrm{kV}$. The typical peak energy tends to increase towards the magnetic midnight. Most of the inverted- $\mathrm{V}$ events occur during magnetically quiet or moderate activity, $K_{p} 1-4$. The usual width of an inverted-V varies from 20 to $40 \mathrm{~km}$ and is comparable to the peak value of the typical meso-scale auroral arc width of $10-20 \mathrm{~km}$. Furthermore, the polar cap potential values estimated from the SuperDARN radar data are always at least three times larger than the inverted- $\mathrm{V}$ acceleration potential drop.
\end{abstract}

Keywords. Ionosphere (Auroral ionosphere; Electric fields and currents; Particle acceleration)

Correspondence to: N. Partamies

(noora.partamies@fmi.fi)

\section{Introduction}

Energies from a few $\mathrm{keV}$ to tens of $\mathrm{keVs}$ within the discrete aurora indicate energization of the magnetospheric particles before they precipitate into the ionosphere. Signatures of the particle acceleration can be seen in the satellite data. Probably the most referred one is the inverted- $\mathrm{V}$, a signature of particle acceleration in a U-shaped potential drop above the auroral arcs. Similar to the arcs, the inverted-V's are thought to be elongated in the east-west direction (along the oval), although the direct measurements by polar orbiting satellites are always meridional cross-sections. At the bottom of the potential drop a field-aligned electric field accelerates electrons towards the ionosphere and ions out of the ionosphere resulting in an upward field-aligned current (FAC). The relation between field-aligned current $j_{\|}$and the potential drop $V$ can be described by the equation $j_{\|}=K\left(V+T_{E} /\right.$ e) (e.g. Janhunen, 1999), where $K$ is an experimental parameter, often referred to as the field-aligned conductance, $T_{E}$ is the electron temperature in the source region, and $e$ is the electron charge. Olsson and Janhunen (2000) demonstrated that the linear relationship between FAC and the acceleration potential drop does not apply nearly as often as was believed in the past, but in most cases the current density is rather independent of the potential. In addition to a quasi-static potential drop, particles can be accelerated by wave-particle interaction, which may also act together with the DC potential drop. The nature of the actual acceleration process, however, is beyond the scope of this paper. The potential structures that accelerate electrons downward and ions upward leave a cavity in the ionospheric plasma density. These cavities have been shown to exist at the altitudes of 2-6 $R_{E}$ (Janhunen et al., 2002) above the auroral oval latitudes. Furthermore, the density depletion regions have been suggested to act as a source for the auroral kilometric radiation (Strangeway et al., 2001). So, various interesting auroral phenomena are related, in one way or another, to the particle acceleration processes.

Published by Copernicus Publications on behalf of the European Geosciences Union. 
The very first report where the term inverted-V was used was published in 1971 by Frank and Ackerson who described different electron acceleration events including an invertedV. Based on a few events they concluded that inverted-V's are substructures occurring during moderate magnetic activity with the $K_{p}$ index ranging from 3 to $4+$. They also found out that the maximum accelerated energy of these structures is of the order of a keV, and their typical duration in the energytime spectrogram is $30-50 \mathrm{~s}$, which corresponds to a distance of about 150-250 km. Bosqued et al. (1985) reported the first statistical study of about 250 inverted-V events as detected by the AUREOL-3 satellite. According to their results, the typical width of the $\mathrm{V}$ structures is $50-100 \mathrm{~km}$ and their peak energies are $1-5 \mathrm{keV}$. These findings suggested that most of the inverted-V's are much wider than many narrow individual auroral arcs $(\sim 100 \mathrm{~m}$, e.g. Borovsky, 1993) captured by high-resolution ground-based instruments. Instead of corresponding single arcs, most of the V-structures have been associated with wider constellations of multiple arcs. However, smaller scale sizes, typically $28-35 \mathrm{~km}$, and an exponential width distribution was reported by Newell et al. (1996).

Based on Defense Meteorological Satellite Program (DMSP) satellite data, an electron acceleration survey by Newell (2000) shows that only $4 \%$ of all of the acceleration events can be called inverted-V's. Furthermore, the invertedV's may not be isolated but rather part of a larger acceleration structure. They classified all Large-Scale Acceleration Events (LSAE) according to their shape in the spectrogram into seven different categories including e.g. flat electron signatures with a constant peak energy, monotonically increasing or decreasing cases, which would correspond to S-shaped potential structures, inverted-V's and forms that resemble inverted-V's. The authors did not comment on the auroral displays corresponding to these different acceleration events. Individual electron spectra within an inverted- $V$ typically has a single peak as a signature of acceleration. Most of these spectra are shown to fit well into an accelerated Maxwellian model spectrum (Olsson and Janhunen, 2000).

There are two main issues considered in this study. The first question is how closely the statistical appearance (i.e. their occurrence frequency as a function of MLT, ILAT, and other parameters) of the inverted-V's can be related to that of the auroral arcs. Another question is whether a relationship can be found between the cross-polar cap potential and the particle acceleration potential above the auroral arcs. The rest of the paper is organised as follows: Sect. 2 describes the data and the event selection criteria, Sect. 3 contains the statistical results of our data set. Defining the widths of the inverted-V's and their comparisons to the ones of the auroral arcs is explained in Sect. 4. Section 5 discusses the observations and compares them both to the previous electron acceleration studies and to the related observations of other auroral phenomena, such as strong ionospheric electric fields, average auroral intensity, upward ion beams, substorm occurrence and plasma density cavities.

\section{Event selection and data}

We define an inverted- $V$ as a $\Lambda$-shaped enhancement of the differential energy flux on the high energy channels on the top of low flux on the lower energies. We also require that the enhancement is strong enough - at least ten times higher than the simultaneous fluxes on the lower energy channels and at high enough energies (at $300 \mathrm{eV}$ or higher) to be easily distinguished from the possible secondary and thermal electron fluxes of the background. The events were also required to be physically large enough, visible in at least three time steps, to form a $\Lambda$ shape. Finally, we looked for symmetric events, whose peak position would not be more than three data points towards either end from the centre of the structure. These requirements are based both on our experience and the previous work by Olsson and Janhunen (2000) and Newell (2000).

The particle detector on board the polar orbiting Fast Auroral SnapshoT (FAST) satellite (Carlson et al., 1998) records energies from $6 \mathrm{eV}$ to $30 \mathrm{keV}$ in 47 channels. The differential energy fluxes (in $\mathrm{eV} / \mathrm{m}^{2} \mathrm{~s} \mathrm{sreV}$ ) are measured separately for pitch angles of $0^{\circ}, 90^{\circ}$ and $180^{\circ}$. In this study, we use all the data for $0^{\circ}$ pitch angle (precipitating) electrons from 1997-2001. FAST orbital period is about $133 \mathrm{~min}$ and thus, it makes about 11 orbits per day and 19760 orbits in 5 years, which is half of the number of the available northern auroral oval crossings. The orbital altitude varies within about $400-4100 \mathrm{~km}$ and the average velocity of the satellite is about $6.9 \mathrm{~km} / \mathrm{s}$. In the FAST quicklook data, the average temporal resolution is about five seconds implying the minimum observable scale size (three time steps) to be about $70 \mathrm{~km}$.

The altitudes of acceleration regions typically range from $1000 \mathrm{~km}$ to about $3 R_{E}$ (e.g. Gorney et al., 1981). Thus, some of the potential structures taking place around the lower end of this altitude range may be only partly recorded or missed by the FAST satellite, which sees only the part of the accelerated precipitation that is located above the orbit.

From the five years worth of data we found 588 inverted$\mathrm{V}$ events in total. An example of a typical inverted- $\mathrm{V}$ in the electron energy-time spectrogram is shown in Fig. 1. This event was observed on 7 May 1999 on the orbit number 10713. It reached the peak energy of about $11 \mathrm{keV}$ and it was observed by FAST for $35 \mathrm{~s}$. This individual event was also examined in FAST high resolution data with the temporal resolution of $0.3 \mathrm{~s}$, and it still showed up as a single, smooth inverted-V.

A subset of 97 excellent events was separated from the full data set. The distributions for all the basic parameters have been checked for both data sets to make sure that their behaviour is similar and thus, our definition of the inverted- $\mathrm{V}$ is strict enough.

The events that were rejected from our event set were typically either not entirely visible due to the upper energy limit of the FAST particle sensor or missing data, highly asymmetric in peak position (by more than three data points towards 


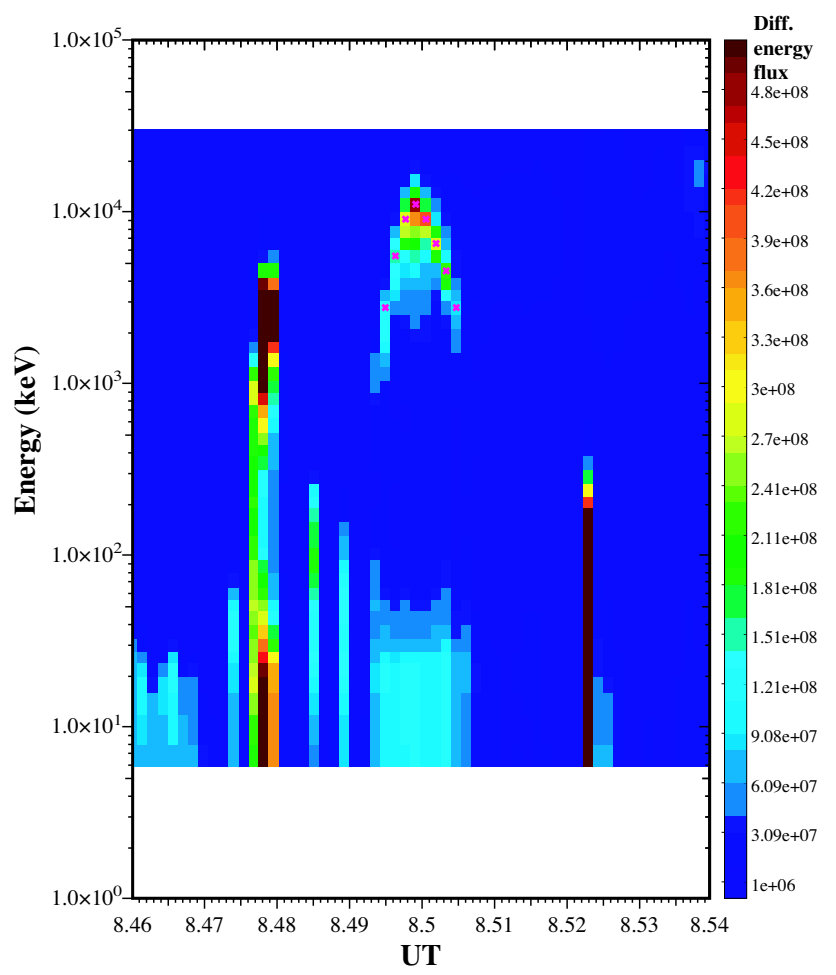

Fig. 1. An example of an inverted-V in an electron spectrogram. Colour coding shows the differential energy flux $\left(\mathrm{eV} /\left(\mathrm{cm}^{2} \mathrm{~s} \mathrm{sreV}\right)\right)$, and pink spots mark the $\mathrm{V}$ structure as defined from the data. Yaxis is energy and $\mathrm{X}$-axis can be given in latitude (GLAT, ILAT), universal time (UT) or magnetic local time (MLT). This event was observed on 7 May 1999 on the orbit number 10713 at 08:27:3608:32:24 UT. FAST saw this inverted-V for about $35 \mathrm{~s}$.

either end), flat or monotonically increasing or decreasing, or had a shape of an upright V.

\section{Statistical results}

Extracting the maximum flux curve (as indicated by the pink crosses in Fig. 1) for each event and plotting these curves for the 97 best events on the top of each other, as shown in Fig. 2, we can examine the shape of the inverted-V's. Averaging over all 97 curves gives the typical shape of these structures as seen in the spectrograms (thick line in Fig. 2). The average curve forms a fairly symmetric and well-defined inverted-V with the peak energy of about $5 \mathrm{keV}$. Peak energies of all of these events vary from a few $\mathrm{keV}$ up to $20 \mathrm{keV}$.

The maximum seasonal occurrence of our events is in November to February, as can be seen from Fig. 3, but there is another maximum (139 events) during the summer months from May to July. In this data set, there are only a few events from the Southern Hemisphere. The curve on the top of the distribution is reconstructed from satellite observations of electron precipitation, according to Fig. 2 by Barth

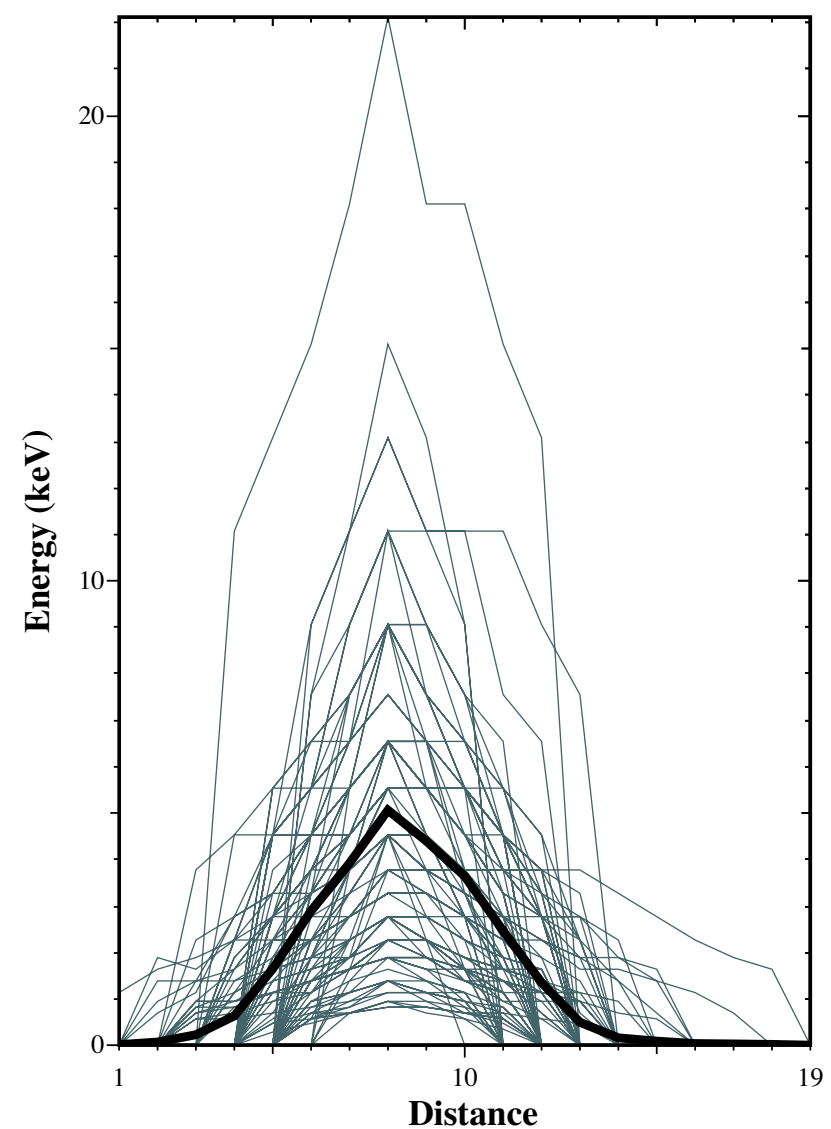

Fig. 2. Energy-distance curve of the best 97 events on the top of each other. One of these curves comes from the pink spots in Fig. 1. The thickest line is an arithmetic average of all curves, i.e. an average shape of the inverted-V's as seen in the electron data. No scaling has been done for these data.

et al. (2004). It shows the seasonal variation of the electron flux ratio of the Northern and Southern Hemispheres. This north-to-south energy flux ratio is high when the Northern Hemisphere energy flux is high (northern winter), and low when the electron precipitation in the Southern Hemisphere is strong (northern summer). The minimum of the flux ratio around the day number 172 (summer solstice) and the maximum around the day number 355 (winter solstice) agree with the main features of the inverted- $\mathrm{V}$ occurrence, except the enhancement in summer. On the other hand, the substorm occurrence as studied by Wang and Lühr (2007) also has a maximum in June in addition to the maximum in December-February. The seasonal maxima in the substorm and inverted- $\mathrm{V}$ occurrence coincide with lowest seasonal values of ionospheric Pedersen conductivity. The only difference we found between the summer maximum events (May to July) and the events during the winter months (November to February) is that the majority of the winter events occur at lower latitudes $\left(65-70^{\circ}\right.$ ILAT), while the summer events are predominantly observed at higher latitudes $\left(75-85^{\circ}\right.$ ILAT). 


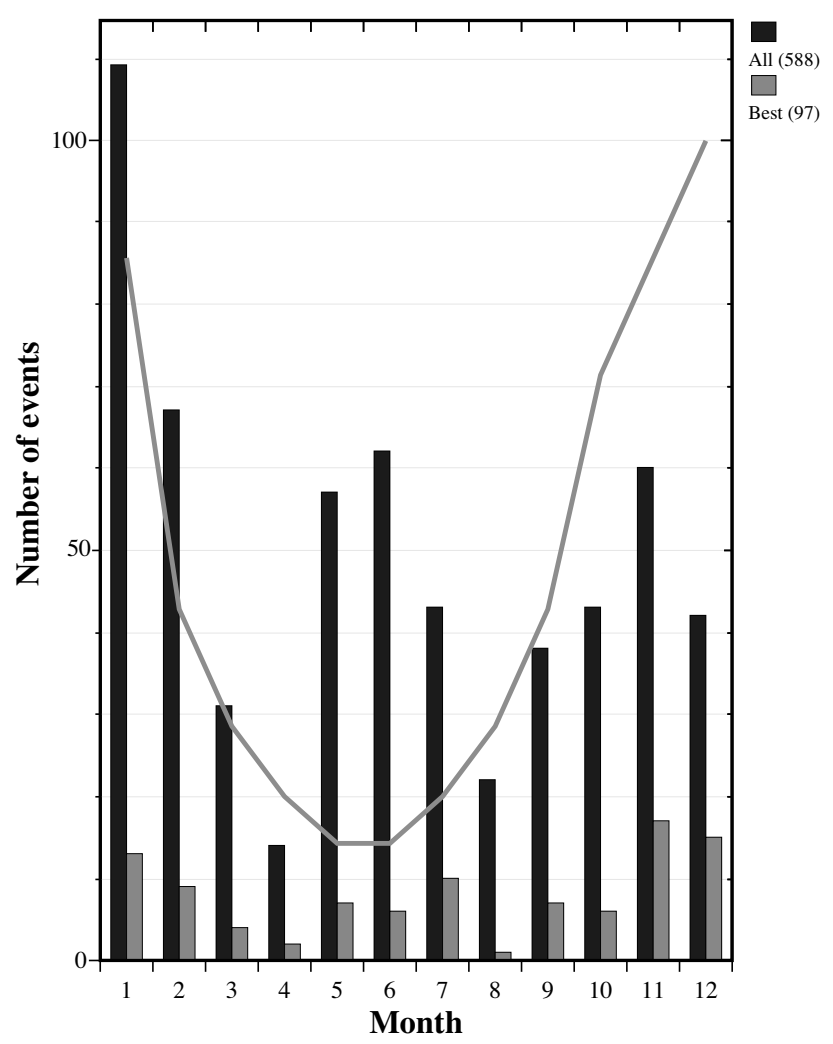

Fig. 3. Seasonal distribution of inverted-V events. Most events are captured during the winter months, but another smaller population shows up during summer. The curve shows the seasonal variation of the electron flux ratio of Northern and Southern Hemispheres (Barth et al., 2004). Inverted-V events of this study have mainly been recorded over the norther auroral region.

The peak energy of the V-structures, i.e. the accelerated maximum energy at the top of the $\Lambda$-shape, is proportional to the acceleration potential drop. This energy value is typically less than $6 \mathrm{keV}(6 \mathrm{kV})$ as shown by Fig. 4. This is in agreement with earlier results by e.g. Olsson et al. (1998), who stated that the potential drops related to auroral arcs range typically up to $5 \mathrm{kV}$, higher values being associated with the onset aurora and Westward Travelling Surge (WTS) type activity (up to $25 \mathrm{kV}$ ). Accelerated energies of $1-5 \mathrm{keV}$ were already suggested by Bosqued et al. (1985). The number of inverted-V's decreases exponentially towards higher peak energies as shown by the curve fitted to the event numbers of the full set (red solid line in Fig. 4). This suggests that within the resolution limits of the observations, events with lower peak energies always occur more frequently. Notice that the first bin of the histogram is left out of the fit because it is biased by the selection criterion that requires the peak energy to be greater than $0.3 \mathrm{keV}$. The satellite altitude may contribute to the observed peak energy if the orbit is too high and crosses the acceleration region so that only a part of the structure can be seen. In this case the peak energy would

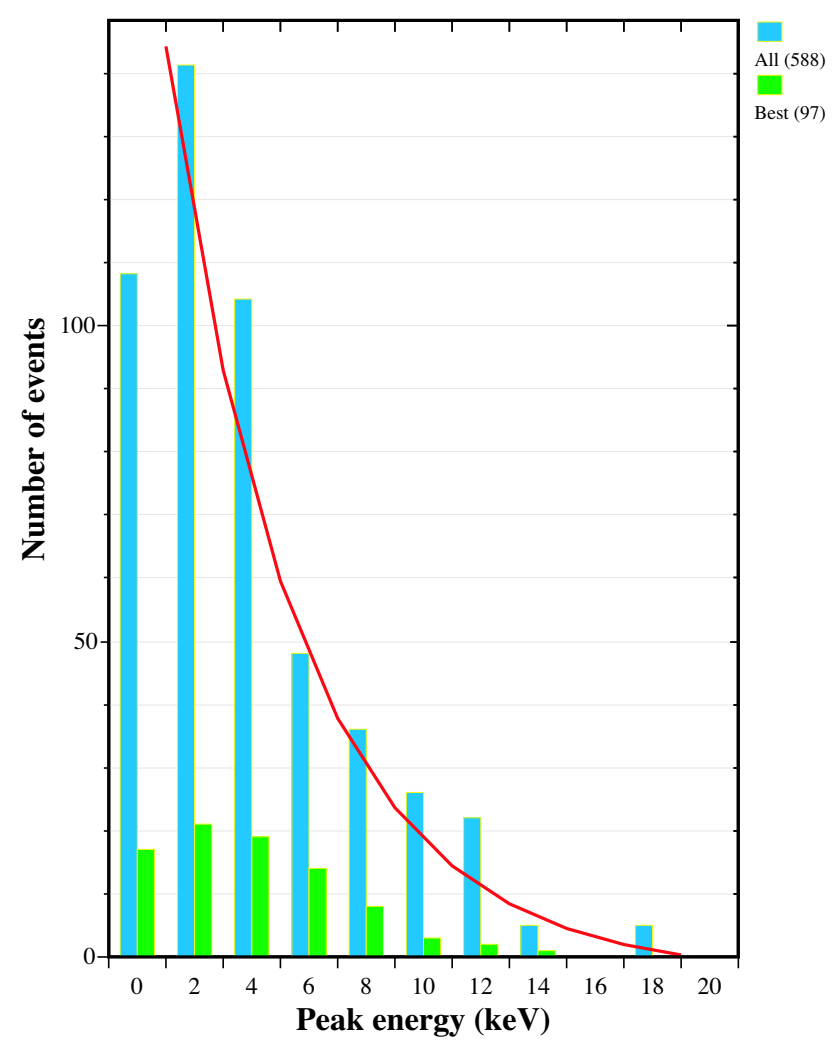

Fig. 4. The distribution of the peak energy observed within the inverted-V's. In most cases, the accelerated energies range from 2 to $4 \mathrm{keV}$. The overlaid red curve shows a fit of an exponential function into the whole data set.

have a tendency to decrease with an increasing altitude of the orbit. Our survey of the FAST altitudes during the inverted$\mathrm{V}$ events (data not shown) revealed no correlation between the peak energy and the spacecraft altitude. Thus, we conclude that our peak energy distribution is not biased by partly recorded events.

Figure 5 shows that the peak energy grows towards the Magnetic Local Time (MLT) midnight, where also the most intense aurora occur. The highest peak energies appear at and around 20:00-24:00 MLT and at and around 70 ILAT. The morning sector in particular is dominated by low acceleration energies. Events at $80^{\circ}$ ILAT or higher typically have low peak energies as well as low energy flux values $\left(<1 \mathrm{~mW} / \mathrm{m}^{2}\right)$. In general, the highest energies appear at the times and places where most of the inverted- $\mathrm{V}$ observations are found: around 21:00 MLT and 70-75 ${ }^{\circ}$ ILAT.

The Magnetic Local Time (MLT) distribution of the inverted-V's in Fig. 6 shows that most of them take place in the evening and pre-midnight sector around 21:0023:00 MLT. This behaviour agrees with that of the overall auroral activity. The MLT distribution in Fig. 6 is normalised by the time FAST spent in each MLT hour bin during the five years. The overlaid curve is the occurrence of about 17000 


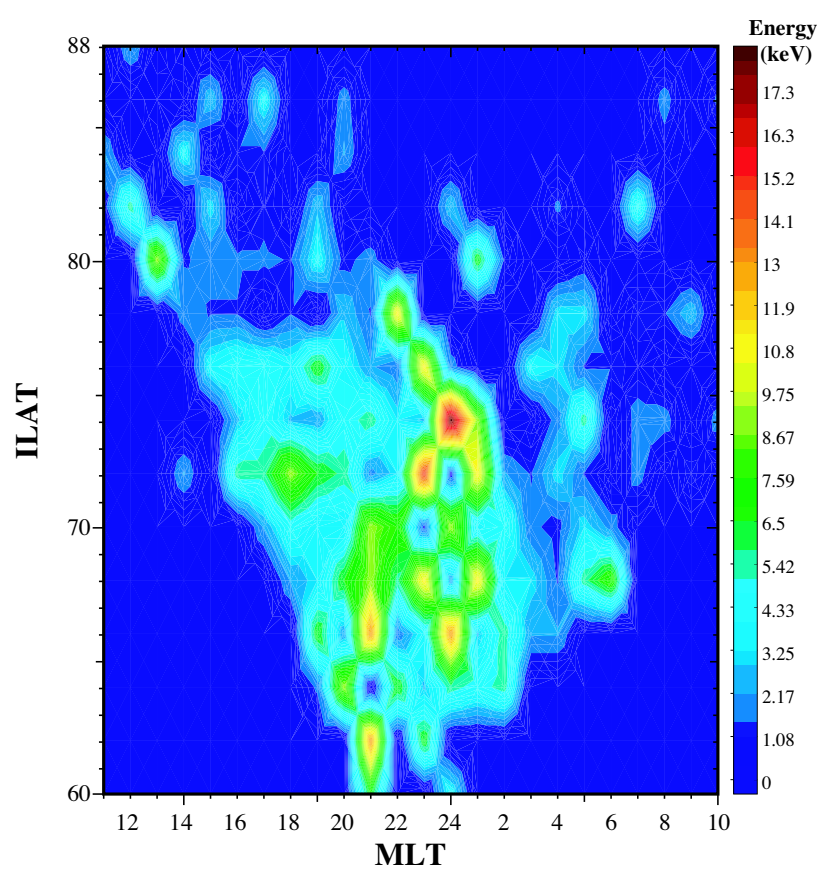

Fig. 5. The distribution of the peak energy (colour-coding) as a function of ILAT and Magnetic Local Time (MLT). Typical invariant latitudes for inverted-V observations are $65-75^{\circ}$. Highest peak energies are usually observed around the magnetic midnight as well as at lower latitudes.

auroral arcs as a function of MLT (Syrjäsuo and Donovan, 2004). The arc occurrence curve, too, has been normalised by the total amount of ASC images taken in each MLT sector. The striking similarity of these two distributions supports the idea that the inverted-V's are the type of acceleration of the auroral arcs.

$K_{p}$ index is a three-hour index of geomagnetic activity. It is generated based on measurements at 12 or 13 stations around the world, and its values range from 0 to 9 . As seen from Fig. 7, the small $K_{p}$ values from one to four are the ones most commonly related to inverted-V's. This distribution is normalised by an equally numbered random set of $K_{p}$ values showing that the most typical of all $K_{p}$ values is one. In addition to the smooth distribution around the small values, there are about ten inverted- $\mathrm{V}$ events taking place during very active conditions, $K_{p} \geq 8$. Provided that the inverted-V's occur together with well-defined auroral arcs, it sounds natural that the typical magnetic activity related to these processes is low or moderate. During high $K_{p}$ values arcs turn into more dynamic aurora, which indicates changes in the particle acceleration structures as well. Higher activity may cause fine structures that are beyond the FAST quicklook data resolution. It may also result in more asymmetric events that, in this study, are not classified as inverted-V's.

The maximum width of an inverted- $\mathrm{V}$, as we call it, is the total length of the structure as seen by the satellite. This is

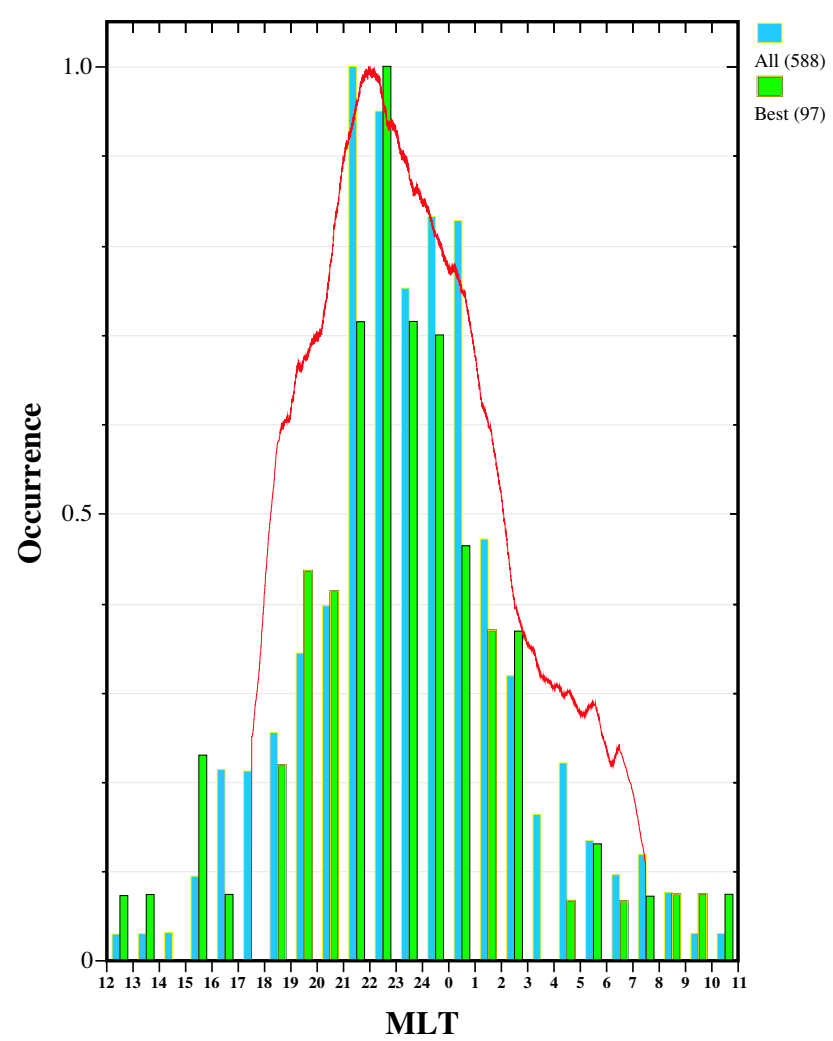

Fig. 6. The distribution of the occurrence of the inverted-V's in MLT shows the maximum occurrence in the pre-midnight sector (21:00-23:00 MLT) where also other auroral phenomena are most often seen. The red curve presents the occurrence of auroral arcs in MLT according to Syrjäsuo and Donovan (2004).

$S=V_{\mathrm{SAT}} \cdot \Delta T$, where $S$ is the maximum length, $V_{\mathrm{SAT}} \cong 7 \mathrm{~km} / \mathrm{s}$ is the average speed of the satellite as it passes an inverted- $\mathrm{V}$ and $\Delta T$ is the time period, during which the $\mathrm{V}$-structure is visible in the satellite data. The typical maximum widths of the structures vary around $130 \mathrm{~km}$ (the distribution not shown), and there is a cut-off around $70-90 \mathrm{~km}$. To be able to identify an inverted-V, it must show up in at least three time steps of the data. In the FAST quicklook data, the average time resolution is $5 \mathrm{~s}$. Consequently, the average speed of the satellite defines the minimum observable inverted- $\mathrm{V}$ length to be about $70 \mathrm{~km}$ (the distance between the first and the third consecutive observation point).

We also determined the cross-polar cap (PC) potential values for each and every inverted- $\mathrm{V}$ event. These potential values come from the Super Dual Auroral Radar Network (SuperDARN) radar (Greenwald et al., 1995) measurements, where spherical harmonics have been fitted to the recorded line-of-sight velocities to produce a smooth convection velocity map and to give an estimate of the cross-polar cap potential (Ruohoniemi and Baker, 1998). The scatter plot in Fig. 8 suggests that the PC potential is always at least three times larger than the potential drop accelerating the 


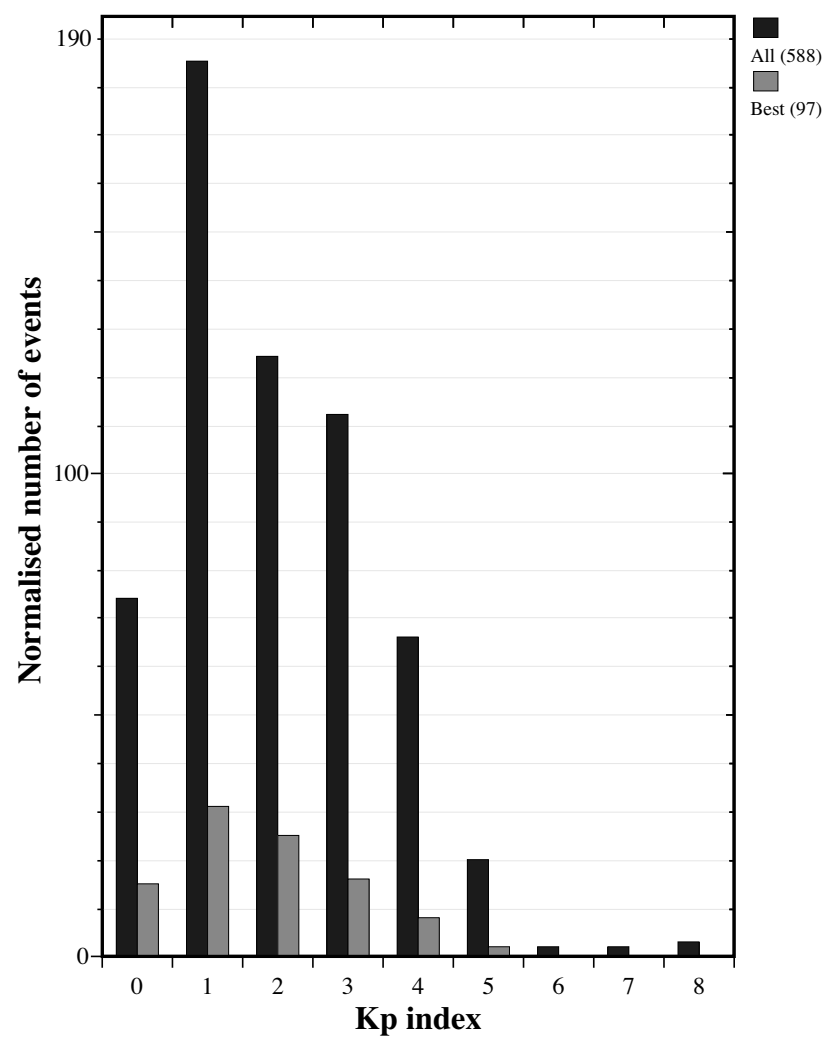

Fig. 7. Distribution of $K_{p}$ index values related to the inverted-V's. The inverted-V's are typically recorded during quiet times or moderate magnetic activity $\left(K_{p} 1-4\right)$. The distribution is divided by the occurrence frequency of each $K_{p}$ value in a randomly selected data set.

inverted-V particles. This finding indicates that the PC potential plays a role in determining the acceleration potentials of auroral arcs. As Fig. 8 also demonstrates, there is a clear cutoff in the PC values around $20 \mathrm{kV}$. The upper end of the $\mathrm{PC}$ range is not as clear but shows that very high potential values are rarely observed together with the inverted- $\mathrm{V}$ type acceleration. There is no reliability check performed for the PC potential values in this figure. In other words, no events were rejected because of, for example, a small amount of actual data points in the convection map. It is not a straightforward task to define when the PC potential estimate is good and thus, we rather rely on the fact that a large number of events averages out the uncertainties.

\section{Peak energy, arc widths and $K_{p}$}

To examine in more detail the relation between the inverted$\mathrm{V}$ peak energy, width (fitted FWHM) and $K_{p}$ index values we divide the data set into sub-groups with peak energies of less than $3 \mathrm{keV}$ (170 events), 3-6 keV (183 events) and over $6 \mathrm{keV}$ (146 events). For these different subsets we plot the distributions of inverted-V widths (Fig. 9) and $K_{p}$ (Fig. 10).

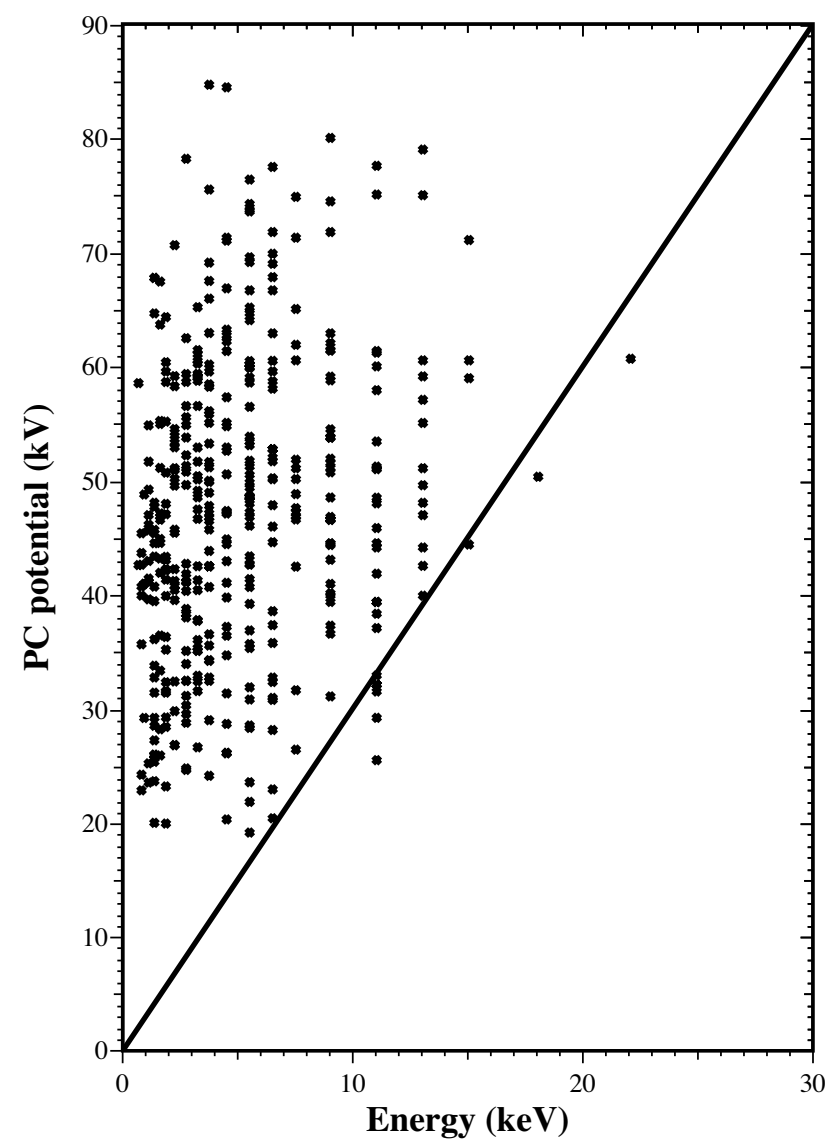

Fig. 8. Cross-polar cap potential measured at the times of the inverted-V observations show that the polar cap potential (Y-axis) is always at least three times larger (solid line) than the peak energy of the inverted-V (X-axis).

According to Fig. 9 most events with the widths less than $60 \mathrm{~km}$ have peak energies less than $6 \mathrm{keV}$ (blue and green bars), while most events with the widths larger than $60 \mathrm{~km}$ are caused by accelerated electrons with the energies greater than $3 \mathrm{keV}$ (green and red bars). So there is slight tendency for smaller structures to be less accelerated. However, no clear division point or clear relation between energies and widths can be found. Any structure size can be associated with any energy range.

The $K_{p}$ index distributions of the same peak energy subsets are plotted in Fig. 10. Similarly to the width distributions of the previous figure, there is no clear $K_{p}$ separation for different energies. But when $K_{p}$ is less than 2 the inverted-V's are often caused by electrons with energies less than $6 \mathrm{keV}$, while for $K_{p}$ higher than 2 the electron energies are usually higher than $3 \mathrm{keV}$. So, higher acceleration energies tend to occur in more active conditions. 


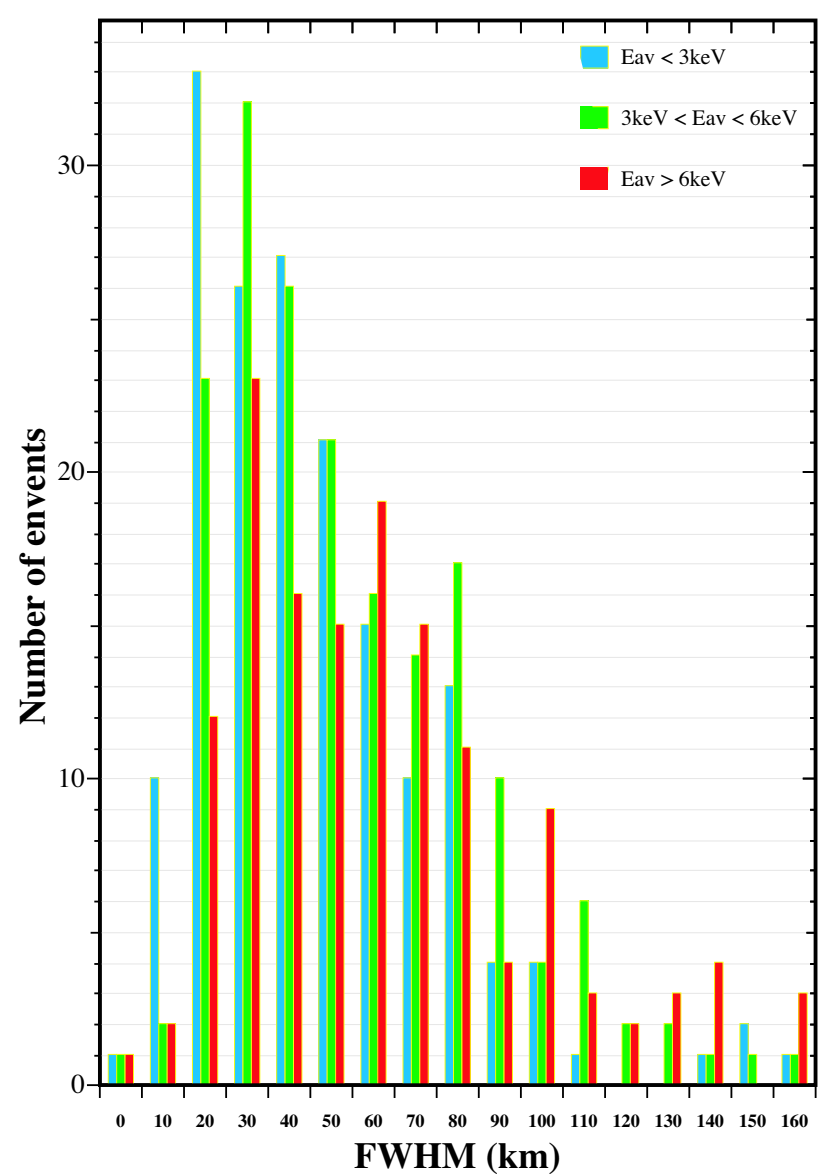

Fig. 9. Distribution of full-width half-maximum (FWHM) values of the inverted-V's for events with peak energies of less than $3 \mathrm{keV}$ (blue), between 3 and $6 \mathrm{keV}$ (green), and more than $6 \mathrm{keV}$ (red).

\section{Gaussian fits}

To be able to compare the inverted- $\mathrm{V}$ scale sizes to the ones of the auroral arcs and to better define the $\mathrm{V}$ widths, we fitted the Gaussian function to the energy flux curve of the V structures. Because the subset of the best events and the whole data set behaved similarly for all other parameters presented in this paper, the whole data set was fitted. An example of an energy flux enhancement corresponding to an inverted- $\mathrm{V}$ is shown in the left panel of Fig. 11. This example event is the same one that was shown in Fig. 1. The energy flux is given as a function of distance (along the FAST trajectory) at the altitude of $100 \mathrm{~km}$.

Prior to the fit, the offsets of the energy flux curves were manually removed and thus, our fitting routine contains only three free parameters: the amplitude of the Gaussian curve $A_{1}$, position of its maximum $A_{2}$ and the full-width halfmaximum (FWHM) value $A_{3}$. The offset was set to be the minimum (background) value of the curve prior to the enhancement in order to keep as many data points as possible, since in many cases there were only a few points in total.

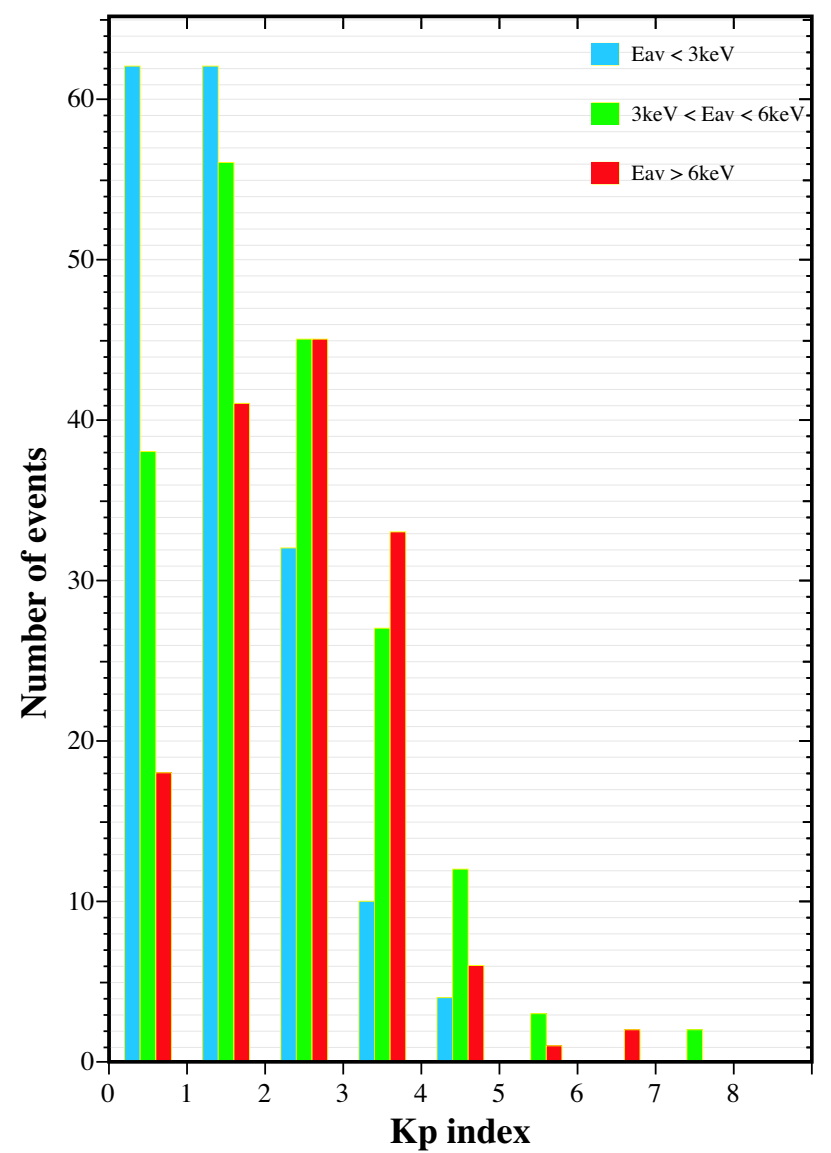

Fig. 10. Distribution of $K_{p}$ index during the inverted-V's for events with peak energies of less than $3 \mathrm{keV}$ (blue), between 3 and $6 \mathrm{keV}$ (green), and more than $6 \mathrm{keV}$ (red).

The mathematical form of the Gaussian function can now be written as $y=A_{1} \exp \left(-\left(x-A_{2}\right)^{2} / 2 A_{3}^{2}\right)$.

For each event, the residual normalised by the energy flux amplitude was defined as $R=\sqrt{\sum\left((y-m)^{2}\right)} / A_{1}$, where $y$ is the Gaussian curve value and $m$ is the corresponding measured energy flux value. We rejected the fits whenever $R \geq 20 \%$ and accepted all fits with $R \leq 10 \%$. This left us with 269 acceptably fitted events. An example of a good fit is shown on the right hand side in Fig. 11, and the distribution of the FWHM values of the fitted inverted-V's can be seen in Fig. 12. The widths in these plots have been determined by using the spacecraft average velocity during each inverted$\mathrm{V}$ event. The typical width of these structures is $20-40 \mathrm{~km}$ and the shape of the distribution slope is approximately exponential. There is again a cutoff on smaller widths that is due to the temporal resolution of the quicklook data and consequently, the minimum observable size. The minimum observable size for the maximum width of the inverted-V's was defined to be about $70 \mathrm{~km}$. Since FWHM values are the efold values of the energy flux curves the minimum observable size is clearly smaller in this examination but cannot 

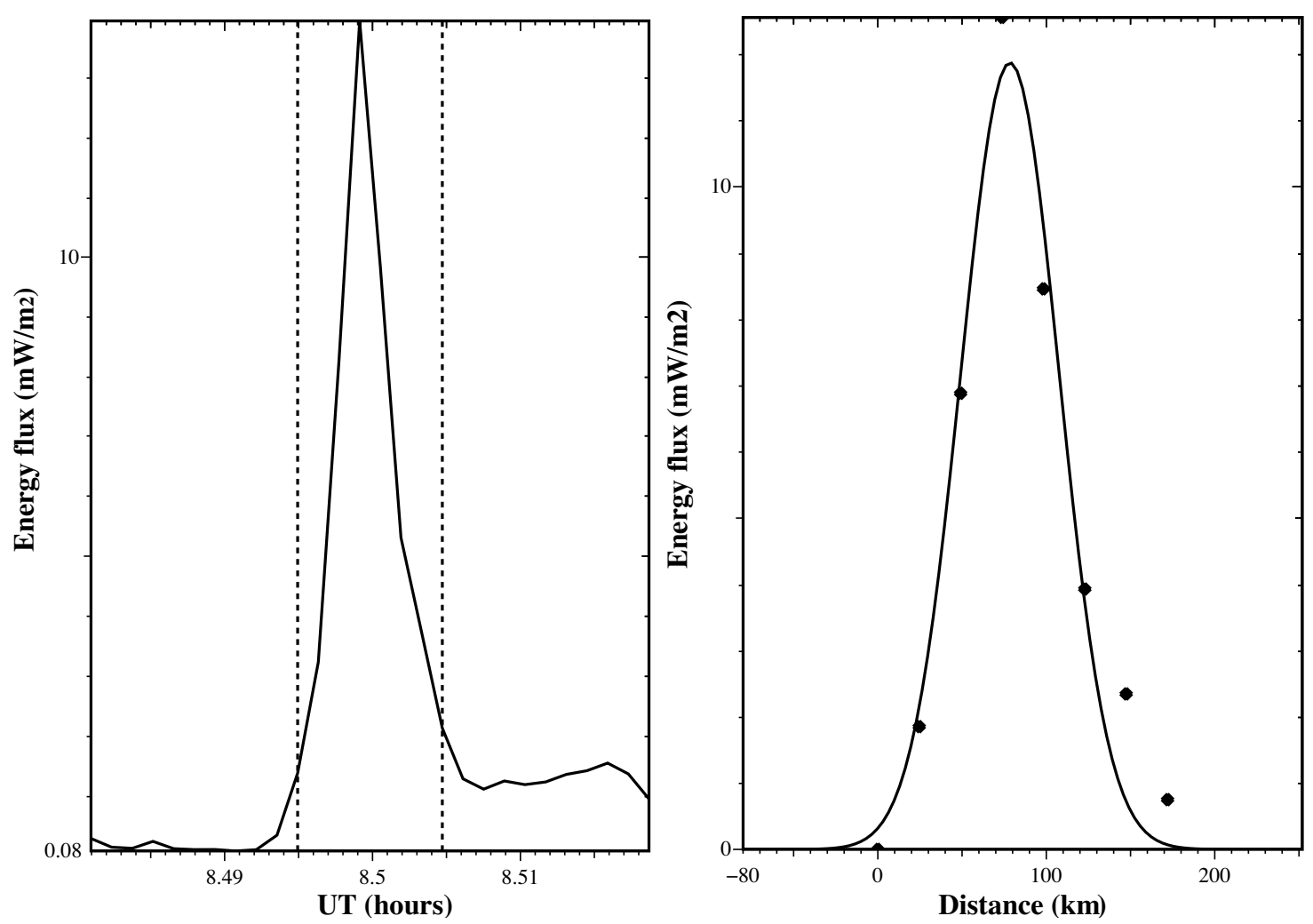

Fig. 11. An example of an energy flux peak within an inverted-V (left hand side) as well as the fitted Gaussian curve to the energy flux (right hand side). The diamonds represent the data points and the vertical lines mark the start and end times of the event corresponding to the first and last pink spots in Fig. 1.

be exactly determined because it depends on the energy flux maximum of each event.

The reference data set in Fig. 12 is the distribution of the widths of the optical auroral arcs as observed by the CANOPUS all-sky camera in Gillam (Knudsen et al., 2001). The fitting of the arc brightness profiles by Knudsen et al. (2001) was performed in a similar manner, and also this optical arc distribution slopes in an exponential way, but the peak appears at somewhat smaller values $(10 \mathrm{~km})$. The inverted-V widths might shift to the smaller values, too, if the highresolution particle data was used in the event selection. The similar behaviour of these two data sets suggest that the meso-scale auroral arcs are the visual traces of the invertedV's seen in the FAST low resolution data. Energy flux is generally a good proxy for auroral brightness, but here the different spatial resolutions of the ground-based imager and the polar orbiting satellite result in slightly different typically observed scale sizes. To be able to tell whether these inverted-V's exactly correspond to single same size arcs or a system of narrower multiple arcs would require conjugate measurements from the ground.

\section{Discussion}

Many things that this statistical study brought up are similar to the occurrence of other auroral phenomena. Observations of upgoing ion beams (Collin et al., 1998; Janhunen et al., 2004) showed a peak occurrence in ILAT $68^{\circ}-71^{\circ}$, which are the average auroral oval latitudes as well as the typical latitudes of the inverted- $\mathrm{V}$ observations of this study. The magnetic local time and the invariant latitude distribution of the inverted-V's are similar to those of the probability of accelerated electrons (Newell et al., 1996) and the average auroral intensity (Liou et al., 1997). One significant difference, however, is that V-structures are also observed at the invariant latitudes of $80^{\circ}$, where the probability of electron acceleration and the average auroral intensity are negligible.

Seasonal variation of other auroral phenomena, such as average auroral luminosity in ultraviolet (Liou et al., 1997), occurrence frequency of upward flowing ion beams (Collin et al., 1998; Janhunen et al., 2004), cosmic radio noise absorption (Yamagishi et al., 1998), ionospheric narrow-band ELF emissions (Satio et al., 1987), occurrence of auroral electromagnetic ion cyclotron (EMIC) waves (Erlandson and Zanetti, 1998), occurrence of auroral kilometric radiation (AKR) (Kumamoto and Oya, 1998), particle acceleration 
(Newell et al., 1996b), intense electric fields (Marklund et al., 1997), and occurrence of auroral particle acceleration events (Liou et al., 2001) behave similarly to the inverted-V's, i.e. decrease towards the summer and increase towards the winter. The plasma density cavities (Janhunen et al., 2002), as well as most of the phenomena mentioned above, are also more frequent in the pre-midnight and midnight sectors and within the latitudes of the auroral zone, where the inverted-V events are most common, too.

Newell et al. (1996) determined the scale size of the accelerated electron events from the DMSP data with $1 \mathrm{~s}$ temporal resolution. They found the typical value for the width of $28-35 \mathrm{~km}\left(\sim 0.3^{\circ} \mathrm{MLAT}\right)$ with a minimum bin size being $0.055^{\circ}$ MLAT. This is in agreement with out findings, but it is likely that in higher resolution data the majority of the observed inverted-V's would be small structures, undetectable in the quicklook data that was used in this study. This would extend the width distribution towards smaller values. Newell et al. (1996) also pointed out that the most promising mechanisms to form auroral arcs with the scale size of $28-35 \mathrm{~km}$ and an exponential width distribution would be shear flows in the central plasma sheet or an ionospheric conductivity feedback mechanism (Borovsky, 1993).

Evans (1994) studied the occurrence of the most intense electron acceleration events $\left(>60 \mathrm{~mW} / \mathrm{m}^{2}\right)$. Their results show that the intense acceleration events are more likely to occur when the $K_{p}$ index is high. This kind of behaviour cannot be seen in our results, where the energy flux values only reach up to about $40 \mathrm{~mW} / \mathrm{m}^{2}$ with the mean value of $7 \mathrm{~mW} / \mathrm{m}^{2}$. Since the inverted-V acceleration is associated with the auroral arcs it is understandable that they are more common during lower magnetic activity than in the heart of the substorm where stable arcs are not observed either. Inside the substorm current wedges, the strong and dynamic currents distort the previously fairly smooth auroral forms, so it is likely that the acceleration potentials and the inverted-V's turn transient and dynamic, too. On the other hand, during a substorm both arcs and inverted-V's may still be observed in the regions outside the expansion bulge itself.

The PC potentials deduced from the SuperDARN measurements showed very coherent behaviour being systematically at least three times larger than the potential difference accelerating electrons into the auroral arcs. The potentials fitted to the SuperDARN data are not always reliable, but the goodness of the fit depends on the number of the data points and the complexity of the current potential pattern. There are several proxies that estimate the cross-polar cap potential based on, for example, $K_{p}$ index or solar wind measurements (Boyle et al., 1997). These kind of proxies would be one way to double-check the potential values. All of the proxies, however, are limited to certain solar wind or magnetic activity conditions and thus, would significantly reduce the number of events in the data set. The best way to confirm the PC potential values from SuperDARN would be to run the procedure that fits the equipotentials to the measured data with

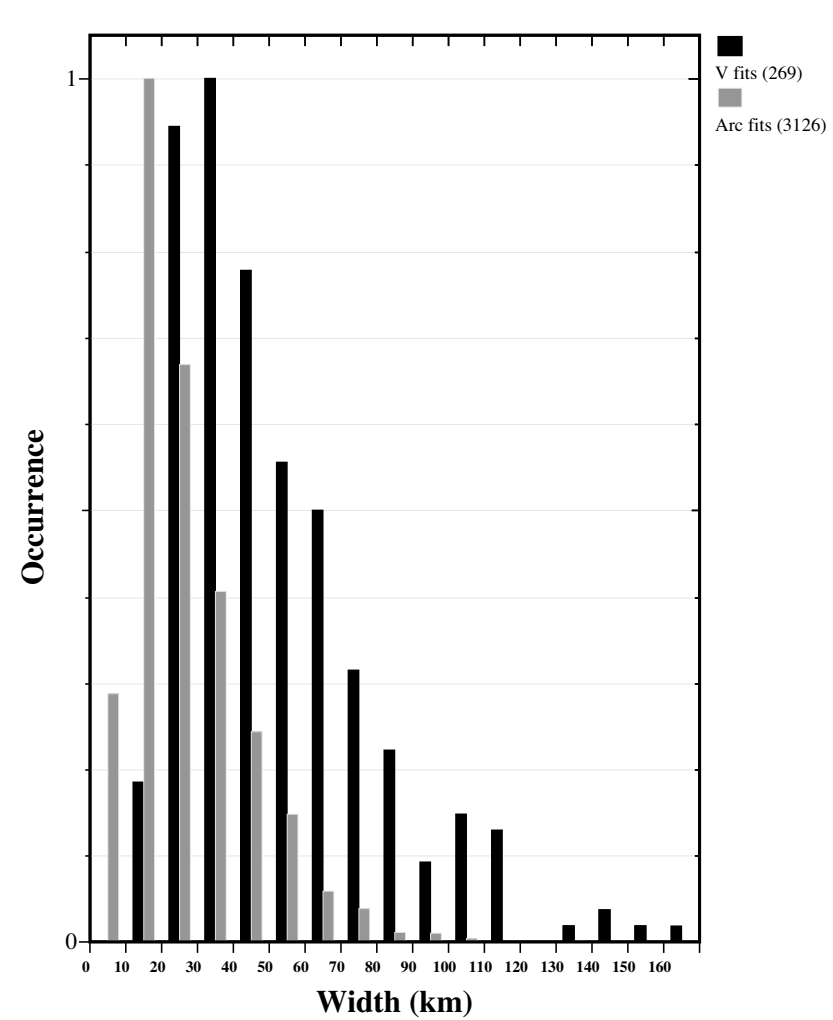

Fig. 12. Distribution of full-width half-maximum (FWHM) values of the inverted-V's as determined from the Gaussian fitting (black bars) compared to the FWHM values of auroral arcs according to Knudsen et al. (2001). Both distributions are similarly shaped but arc widths peak on smaller values.

random solar wind parameters. If this did not change the potential values much, then the measured data points would have a bigger effect in the resulting cross-polar cap potential than the model functions of the fit and the result would be reliable. If the random solar wind data radically changed the potential values, the solar wind driving would then have a bigger role than the measured ionospheric velocities and the coverage of the actual measurements would probably not be good enough for determining the cross-polar cap potential. This, however, is left aside as a future task. Furthermore, according to Hairston et al. (1998), the polar cap potential values larger than $40 \mathrm{kV}$ indicate a two-cell convection pattern, while values smaller than that imply a weak coupling between the magnetosphere and the solar wind. In our data set about one third of the potential values are smaller than $40 \mathrm{kV}$, and all of the potentials are smaller than $90 \mathrm{kV}$, which according to Hairston et al. leaves out the possibility of geomagnetic storm conditions. The most reliable PC potentials are related to the two-cell pattern and thus, at least two thirds of our events should be rather well-defined.

The whole discussion about arc-related potential drops and cross-polar cap potentials is included here to show that although single arcs cannot be reliably mapped to the 
magnetosphere, there is still something in the magnetosphere that controls the smaller-scale auroral processes. The polar cap potential, or the dawn-dusk electric field, seems to set an upper limit to the arc-related acceleration potential.

\section{Conclusions}

In this paper, we have studied five years (1997-2001) of electron spectrograms from FAST satellite to find as many well-defined inverted-V events as possible. With the total number of inverted-V's of 588, we can conclude that these events mainly occur at the auroral oval latitudes $\left(65^{\circ}-75^{\circ}\right.$ ILAT), predominantly during the winter months (November to February) and typically during the pre-midnight MLT hours (21:00-23:00 MLT). Minor populations occurring during the summer months (May to July) or at invariant latitudes higher than $80^{\circ}$ were also observed, but whether these events are different from the main population remains unresolved. Although there are many auroral phenomena that may behave statistically similarly to our inverted-V's, the most important is the agreement with the auroral arc observations from the ground: in addition to the very similar occurrence in MLT, we also found that the widths of the arcs and the inverted-V's are mainly of the same size.

The typical accelerated energies of the inverted-V's are small, mainly less than $6 \mathrm{keV}$. This would correspond to the accelerating potential differences of less than $6 \mathrm{kV}$ being in a good agreement with earlier observations. However, the exponentially decreasing number of events as a function of increasing energy suggests that the lower the energy threshold is in the selection process the more events may be found in the low energy range. Most the $\mathrm{V}$ events appear during magnetically quiet or moderately active conditions with $K_{p} 1-4$. However, there is a tendency for (smaller) larger structures to be caused by (lower) higher energy electrons, or (quiet) active time events to be related to (less) more energetic precipitation.

The most interesting finding of this paper is the link between the ionospheric and magnetospheric processes. Namely, the cross-polar cap potential seems to be always at least three times greater than the acceleration potential drop of the inverted-V's. This means that the polar cap potential, or the dawn-dusk electric field plays a role in controlling the small-scale processes in the ionosphere.

Acknowledgements. The work by N. Partamies was supported by Alberta Ingenuity Fund, and the Academy of Finland. The authors thank Robin Barnes for providing the SuperDARN polar cap potential data.

Topical Editor M. Pinnock thanks J. D. Menietti and J. Borovsky for their help in evaluating this paper.

\section{References}

Barth, C. A., Baker, D. N., and Bailey, S. M.: Seasonal variation of auroral electron precipitation, Geophys. Res. Lett., 31, L04809, doi:10.1029/2003GL018892, 2004.

Borovsky, J. E.: Auroral arc thicknesses as predicted by various theories, J. Geophys. Res., 98, 6101-6138, 1993.

Bosqued, J. M., Maurel, C., Reme, H., Sauvaud, J. A., and Kovrazhkin, R. A.: Statistical study on inverted-V events - A comparison between experiment and theory, Adv. Space Res., 5, 135-138, 1985.

Boyle, C. B., Reiff, P. H., and Hairston, M. R.: Empirical polar cap potentials, J. Geophys. Res., 102, 111-125, 1997.

Carlson, C. W., Pfaff, R. F., and Watzin, J. G.: The Fast Auroral Snapshot mission, Geophys. Res. Lett., 25, 2013-2016, 1998.

Collin, H. L., Peterson, W. K., Lennartsson, O. W., and Drake, J. F.: The seasonal variation of auroral ion beams, Geophys. Res. Lett., 25, 4071-4074, 1998.

Erlandson, R. E. and Zanetti, L. J.: A statistical study of auroral electromagnetic ion cyclotron waves, J. Geophys. Res., 103, 4627-4636, 1998

Evans, D. S.: A study of intense auroral electron precipitation events, Proceedings of the Second Space Environment Center Users Conference, Boulder, CO, 1994.

Frank, L. A. and Ackerson, K. L.: Observations of charged particle precipitation into the auroral zone, J. Geophys. Res., 76, 36123643, 1971.

Gorney, D. J., Clarke. A., Croley, D., Fennell, F., Luhman, J., and Mizera, P.: The distribution of ion beams and conics below 8000 km, J. Geophys. Res., 86, 83-89, 1981.

Greenwald, R. A., Baker, K. B., Dudeney, J. R., Pinnock, M., Jones, T. B., Thomas, E. C., Villain, J.-P., Cerisier, J.-C., Senior, C., Hanuise, C., Hunsucker, R. D., Sofko, G., Koehler, J., Nielsen, E., Pellinen, R., Walker, A. D. M., Sato, N., and Yamagishi, H.: DARN/SuperDARN, A global view of the dynamics of highlatitude convection, Space Sci. Rev., 71, 761-796, 1995.

Hairston, M. R., Heelis, R. A., and Rich, F. J.: Analysis of the ionospheric cross polar cap potential drop using DMSP data during the National Space Weather Program study period, J. Geophys. Res., 103, 26337-26 348, 1998.

Janhunen, P.: On the current-voltage relationship in fluid theory, Ann. Geophys., 17, 11-26, 1999, http://www.ann-geophys.net/17/11/1999/.

Janhunen, P., Olsson, A., and Laakso, H.: Altitude dependence of plasma density in the auroral zone, Ann. Geophys., 20, 17431750, 2002, http://www.ann-geophys.net/20/1743/2002/.

Janhunen, P., Olsson, A., and Laakso, H.: The occurrence frequency of auroral potential structures and electric fields as a function of altitude using Polar/EFI data, Ann. Geophys., 22, 951-970, 2004 , http://www.ann-geophys.net/22/951/2004/.

Knudsen, D. J., Donovan, E. F., Cogger, L. L., Jackel, B., and Shaw, W. D.: Width and structure of mesoscale optical auroral arcs, Geophys. Res. Lett., 28, 705-708, 2001.

Kumamoto, A. and Oya, H.: Asymmetry of occurrence-frequency and intensity of AKR between summer polar region and winter polar region sources, Geophys. Res. Lett., 25, 2369-2372, 1998.

Liou, K., Newell, P. T., Meng, C.-I., Lui, A. T. Y., Brittnacher, M., and Parks, G.: Synoptic auroral distribution: A survey using Po- 
lar ultraviolet imagery, J. Geophys. Res., 102, 27 197-27 206, 1997.

Liou, K., Newell, P. T., and Meng, C. I.: Seasonal effects on auroral particle acceleration and precipitation, J. Geophys. Res., 106, 5531-5542, 2001.

Marklund, G., Karlsson, T., and Clemmons, J.: On low altitude particle acceleration and intense electric fields and their relationship to black aurora, J. Geophys. Res., 102, 17 509-17 522, 1997.

Newell, P., Lyons, K., and Meng, C.: A large survey of electron acceleration events, J. Geophys. Res., 101, 2599-2614, 1996.

Newell, P., Meng, C., and Wing, S.: Relation to solar activity of intense aurorae in sunlight and in darkness, Nature, 393, 342344, 1996.

Newell, P.: Reconsidering the inverted-V particle signature: Relative frequency of large-scale electron acceleration events, J. Geophys. Res., 105, 15 779-15 794, 2000.

Olsson, A., Andersson, L., Eriksson, A. I., Clemmons, J., Erlandsson, R. E., Reeves, G., Hughes, T., and Murphee, J. S.: Freja studies of the current-voltage relation in substorm-related events, J. Geophys. Res., 103, 4285-4301, 1998.

Olsson, A. and Janhunen, P.: A statistical study of nightside inverted-V events using Freja electron data: implications for the current-voltage relationship, J. Atmos. Terr. Phys., 62, 81-92, 2000.
Ruohoniemi, J. M. and Baker, K. B.: Large-scale imaging of highlatitude convection with Super Dual Auroral Radar Network HF radar observations, J. Geophys. Res., 103, 20 797-20 811, 1998.

Satio, H., Yoshino, T., and Sato, N.: Narrow-banded ELF emissions over the Southern polar region, Planet. Space Sci., 35, 745-752, 1987.

Strangeway, R. J., Ergun, R. E., Carlson, C. W., McFadden, J. P., Delory, G. T., and Pritchett, E. L.: Accelerated electrons as the source of auroral kilometric radiation, Phys. Chem. Earth, 26(13), 145-149, 2001.

Syrjäsuo, M. T. and Donovan, E. F.: Diurnal auroral occurrence statistics obtained via machine vision, Ann. Geophys., 22, 1103 1113, 2004, http://www.ann-geophys.net/22/1103/2004/.

Yamagishi, H., Fujita, Y., Sato, N., Stauning, P., Nishino, M., and Makita, K.: Conjugate features of auroras observed by TV cameras and imaging riometers at auroral zone and polar cap conjugate-pair stations, in: Polar cap boundary phenomena, edited by: Moen, J., Egeland, A., and Lockwood, M., 289-300, Kluwer Acad., Norwell, Mass., 1998.

Wang, H. and Lühr, H.: Seasonal-longitudinal variation of substorm occurrence frequency: Evidence for ionospheric control, Geophys. Res. Lett., 34, L07104, doi:10.1029/2007GL029423, 2007. 